\title{
Practical Guidance of the GTH Haemophilia Board on the Use of Emicizumab in Patients with Haemophilia A
}

\author{
Katharina Holstein ${ }^{1}$ Manuela Albisetti ${ }^{2}$ Christoph Bidlingmaier ${ }^{3}$ Susan Halimeh ${ }^{4}$ Sabine Heine ${ }^{5}$ \\ Robert Klamroth $^{6}$ Christoph Königs ${ }^{7}$ Karin Kurnik ${ }^{8}$ Christoph Male $^{9}$ Johannes Oldenburg ${ }^{10}$ \\ Werner Streif ${ }^{11}$ Cornelia Wermes ${ }^{12}$ Carmen Escuriola-Ettingshausen ${ }^{13}$ and on behalf of the 'Ständige \\ Kommission Hämophilie' (Haemophilia board) of the German, Swiss Austrian Society for Thrombosis \\ Haemostasis Research (GTH)
}

${ }^{1}$ Haematology and Clinical Oncology, University Medical Center Hamburg-Eppendorf, Hamburg, Germany

2 Division of Hematology, University Children's Hospital, Zurich, Switzerland

${ }^{3}$ Centre for Development and Complex Chronic Diseases in Children (iSPZHauner), Department of Paediatrics, Paediatric Haemophilia Centre, LMU Munich, Munich, Germany

${ }^{4}$ Coagulation Center Rhein-Ruhr, Duisburg, Germany

${ }^{5}$ Department of Paediatric Oncology/Haematology, Saarland

University Hospital, Homburg, Germany

${ }^{6}$ Internal Medicine, Angiology and Haemostaseology, Haemophilia Centre, Vivantes Hospital Friedrichshain, Berlin, Germany

${ }^{7}$ Department of Paediatrics and Adolescent Medicine, University

Hospital Frankfurt, Goethe University, Frankfurt, Germany

8 Paediatric Haemophilia Centre, Department of Paediatrics, LMU

Munich, Munich, Germany

${ }^{9}$ Department of Paediatrics, Medical University of Vienna, Vienna, Austria

10 Institute for Experimental Haematology and Transfusion Medicine, University Hospital Bonn, Bonn, Germany

${ }^{11}$ Department of Paediatrics 1, Medical University of Innsbruck (MUI), Innsbruck, Austria

12 Haemophilia Center, Werlhof-Institute, Hannover, Germany

13 Haemophilia Center Rhine Main, HZRM, Mörfelden-Walldorf, Germany
Address for correspondence Dr. Katharina Holstein, Haemophilia Centre, II. Medical Department, University Medical Center HamburgEppendorf, Martinistr. 52, 20246, Hamburg (e-mail: k.holstein@uke.de).

Hämostaseologie 2020;40:561-571.
Abstract
Keywords
- Emicizumab
- haemophilia A
- inhibitors
- prophylaxis

Emicizumab has been approved for bleeding prophylaxis in patients with haemophilia $A$ (PWHAs) with or without inhibitors. Because of substantial differences between factor VIII (FVIII) and Emicizumab, the 'Ständige Kommission Hämophilie' of the German, Austrian, Swiss Society for Thrombosis and Haemostasis Research (GTH) established a practical guidance for the use of Emicizumab in PWHAs. A systematic literature research was conducted in PubMed. Based on this and on personal experience, this practical guidance has been developed. Each single statement has been discussed among members of the 'Ständige Kommission Hämophilie' and revised accordingly. The final set of recommendations has been approved by all authors analogous to the Delphi method. This practical guidance is provided for physicians treating PWHAswith regard to general aspects, patient education, bleeding treatment, surgery, use of Emicizumab in previously untreated patients (PUPs), patients with newly diagnosed inhibitors and elderly patients. Patients should be treated in expert centres and adequate laboratory tests to monitor Emicizumab received

November 11, 2019

accepted after revision

February 25, 2020 (c) 2020 Georg Thieme Verlag KC Stuttgart · New York
DOI https://doi.org/

10.1055/a-1127-6476.

ISSN 0720-9355. 
levels, FVIII replacement and inhibitors should be available. Early experience of immune tolerance induction protocols integrating Emicizumab is reviewed, and the limited experience in PUPs and very young children is described. So far, no thromboembolic complications have been reported with the concomitant use of FVIII or recombinant activated FVII for bleeding treatment or surgery. Activated prothrombin complex concentrate doses of $>100 \mathrm{U} / \mathrm{kg}$ for $>24$ hours should be avoided whenever possible because of the high risk of thrombosis and/or thrombotic microangiopathy. In conclusion, this study is designed to support haemophilia physicians using Emicizumab in physicians treating hemophilia and using (PWHAs). With further post-marketing experience and trials, regular updates are necessary.

\section{Zusammenfassung}

Schlüsselwörter

- Emicizumab

- Haemophilia A

- Inhibitoren

- Prophylaxe
Einleitung Emicizumab, ein bispezischer Antikörper, der wie aktivierter Faktor VIII (FVIII) wirkt, ist zugelassen zur Blutungsprophylaxe bei Patienten mit Hämophilie A ohne und mit FVIII-Inhibitor. Aufgrund substanzieller Unterschiede zwischen Emicizumab und FVIII hat die "Ständige Kommission Hämophilie“ der deutschen, österreichischen und schweizerischen Gesellschaft für Thrombose- und Hämostaseforschung (GTH) beschlossen, einen praxisorientierten Leitfaden für den Einsatz von Emicizumab bei Patienten mit Hämophilie A (PWHA) zu entwickeln.

Methoden Daten aus der Literatur (systematische Literaturrecherche in PubMed) und persönliche Erfahrungen wurden zusammengetragen und daraus Empfehlungen entwickelt. Diese wurden in der „Ständigen Kommission Hämophilie“ diskutiert und entsprechend revidiert. Die finalen Empfehlungen wurden von allen Autoren mittels Delphi-Abstimmung freigegeben.

Ergebnisse Die Empfehlungen beziehen sich auf Patienten ohne und mit FVIIIInhibitor und beinhalten generelle Aspekte, Patientenaufklärung, Blutungsbehandlung, Operationen, Anwendung von Emicizumab bei zuvor unbehandelten Patienten (PUPs), Patienten mit neudiagnostizierten Inhibitoren und älteren Patienten. Patienten sollten in spezialisierten Behandlungszentren behandelt werden und adäquate Labortests zum Monitoring von Emicizumab, einer Faktor VIII-Substitution oder von FVIII-Inhibitoren sollten zur Verfügung stehen. Erste Erfahrungen mit Protokollen zu Immuntoleranz -Induktion in Kombination mit Emicizumab und die begrenzte Erfahrung mit sehr jungen Kindern wird berichtet. Bis jetzt wurden keine thrombotischen Komplikationen bei der zusätzlichen Blutungsbehandlung mit rFVIIa oder FVIII berichtet. aPCC-Dosierungen von $>100 \mathrm{U} / \mathrm{kg}$ über $>24$ Stunden müssen vermieden werden, wegen des Risikos von Thrombosen und/oder thrombotischer Mikroangiopathien.

Schlussfolgerung Die Empfehlungen wurden anhand der aktuell verfügbaren Erfahrung entwickelt als Leitfaden für Hämophiliebehandler, die Emicizumab bei PWHA einsetzen. Mit weiterer Erfahrung und neuen Studien ist ein regelmäßiges Überarbeiten der Empfehlungen erforderlich.

\section{Introduction}

Emicizumab is a recombinant bispecific antibody with humanised binding sites to activated factor IX (FIXa) and factor X(FX), designed to mimic cofactor activity of factor VIII (FVIII). ${ }^{1}$ It was developed for bleeding prophylaxis in patients with haemophilia A (PWHAs) with and without inhibitors. ${ }^{2}$

For full haemostatic activity, Emicizumab needs phosphatidylserine-exposed phospholipids at the site of injury. ${ }^{1}$ The route of administration is subcutaneous, and the half-life is approximately 4 to 5 weeks. ${ }^{3}$ Pivotal clinical studies have been published in 2017 and $2018^{4,5}$ leading to licensure of Emicizumab for bleeding prophylaxis in PWHAs with inhibitors in November 2017 by the U.S. Food and Drug Administration (FDA) and in February 2018 by the European Medicines Agency (EMA), and for PWHAs without inhibitors in October 2018 by FDA and in March 2019 by EMA.

The mode of action, laboratory testing, potential interactions and adverse events are substantially different from FVIII and require an adaption of management of 
bleeds/surgery and laboratory monitoring. ${ }^{4,6}$ Therefore, the 'Ständige Kommission Hämophilie' of the German, Austrian, Swiss Society for Thrombosis and Haemostasis Research (GTH) decided to develop a practical guidance document with recommendations and precautions for the use of Emicizumab in PWHAs.

\section{Methods}

A literature search in PubMed last updated on October 16, 2019, according to the PRISMA guidelines, ${ }^{7}$ was performed using the search terms 'haemophilia' and 'Emicizumab', revealing 108 publications. Additionally, congress abstracts of American Society of Haematology, International Society on Thrombosis and Haemostasis and European Association of Haemophilia and Allied Disorders congresses were screened. The search history is shown in -Fig. 1. A final selection of 31 papers, focusing on treatment with Emicizumab, efficacy, safety and laboratory testing, was included into the literature review. The recommendations were drafted by the authors and presented at the meeting of the GTH-Haemophilia Board 'Ständige Kommission Hämophilie' at the annual congress of GTH in February 2019. Critical points were discussed extensively among the active members of the board. A revised draft of recommendations was discussed among the authors in a telephone conference as well as few statements, in which complete consensus was not reached, decided by using an electronic survey. A final set of recommendations was circulated to all 13 authors for approval via an online survey analogous to the Delphi

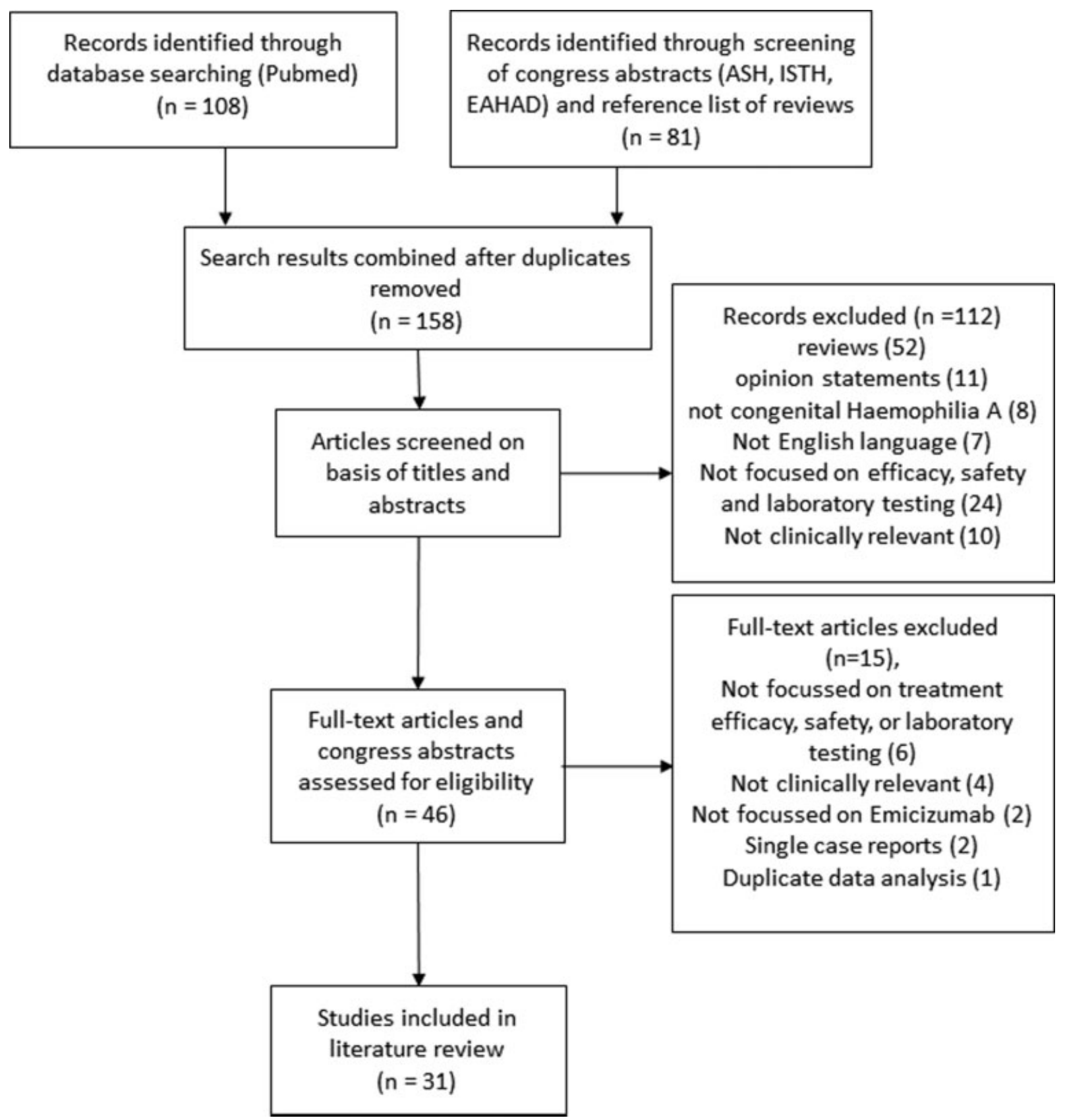

Fig. 1 Search history for the systematic literature review following PRISMA guidelines. 
method with a 5-point Likert scale with rating as follows: strong agreement, agreement, limited agreement, concern, and no agreement. Consensus was determined if a statement reached $>80 \%$ responses indicating 'strong agreement' or 'agreement' according to one suggested threshold for the Delphi method. ${ }^{8}$ All 13 authors responded to the survey. In -Table 1 the final recommendations and the proportion of agreement are displayed. The final version of the revised manuscript was circulated and approved by all authors.

\section{General Aspects}

Emicizumabls Licensed for Prophylactic Treatment in Patients with Severe Haemophilia A with and without Inhibitors of all Ages

- The decision to use Emicizumab as a prophylactic approach in PWHAs has to be made on an individual basis, considering the individual situation of the patient (e.g., patients with persistent FVIII inhibitors, venous access, bleeding phenotype) and risk factors.

- There is limited experience concerning the use of Emicizumab in PWHAs after successful immune tolerance induction (ITI) in PUPs, small children, particularly newborns, children $<2$ years and elderly patients $>65$ years of age.

- After a loading dose of Emicizumab of $3 \mathrm{mg} / \mathrm{kg}$ subcutaneous (sc) per week for 4 weeks, a maintenance dose of $1.5 \mathrm{mg} / \mathrm{kg}$ sc once weekly (qw), $3 \mathrm{mg} / \mathrm{kg}$ sc once every 2 weeks (q2w) or $6 \mathrm{mg} / \mathrm{kg}$ sc once every 4 weeks ( $\mathrm{q} 4 \mathrm{w}$ ) is approved. ${ }^{4,5,9}$

- The choice of the dosing regimen can be based on clinical criteria, patient's preference and vial size.

\section{Recommendations}

1.Treatment with Emicizumab should be supervised by experienced Haemophilia centres.

(a) The medical staff should be experienced in diagnosis and treatment of PWHAs with and without inhibitors.

(b) The medical staff has to be educated in mode of action, risks and potential side effects of Emicizumab.

(c) 24-hour emergency cover and access to adequate laboratory tests to monitor patients under Emicizumab as well as rescue therapies have to be provided.

(d) Other health care professionals (e.g., general practitioner, physical therapist, dentist) involved in the patients' treatment have to be informed, e.g., by a discharge letter and the Emicizumab emergency card.

(e) If applicable, patients should be included into clinical trials (interventional trial, observational studies or registries)

\section{Supporting Data}

\section{Efficacy and Safety}

The phase 3 HAVEN study program evaluated efficacy and safety in PWHAs with and without inhibitors. ${ }^{4,5,9,10}$ For PWHAs with inhibitors aged 12 years or older (HAVEN 1), the annualised rate of treated bleeds (ABR) was 2.9 (95\% confidence interval $[\mathrm{CI}]: 1.7-5.0)$ in the group randomly assigned to Emicizumab prophylaxis $(n=35)$ compared with 23.3 (95\% CI: 12.3-43.9) in those assigned to on-demand treatment $(n=18), p<0.001$. In total, $63 \%$ of patients with Emicizumab prophylaxis experienced no bleeds compared with $6 \%$ of patients without prophylaxis. An updated analysis revealed further reduction of ABR in the second and third 24week observation periods and a further increase in proportion of patients without any treated bleeds. ${ }^{11}$ Most frequent side effects were injection-site skin reactions in 15 to $22 \%$ of participants. These were usually mild and self-limiting. Thrombotic microangiopathy (TMA) and thrombosis were reported in two cases each in the primary analysis and another case of TMA after data cut-off for primary analysis. These events occurred in patients receiving multiple dosing of activated prothrombin complex concentrate (aPCC) for breakthrough bleeding ( $>100 \mathrm{IU} / \mathrm{kg}$ for $>24$ hours) ${ }^{4}$

Another case of TMA with concomitant use of aPCC was reported post-marketing, so as of September 30, four TMA cases were reported out of $>5,400$ patients treated. In addition to the two cases in HAVEN 1, seven more cases of thrombotic events (without TMA) were reported, all seven occurred with aPCC $<100 \mathrm{U} / \mathrm{kg} / 24 \mathrm{~h}$ or with Emicizumab alone, but patients had underlying risk factors like previous pulmonary embolism or arterial hypertension. Up to September 30, 2019, 17 fatalities were reported in clinical trials, compassionate use, expanded and post-marketing access, but none was judged to be related to Emicizumab. ${ }^{12}$ No data on mortality rate of PWHAs with inhibitors without Emicizumab prophylaxis are available for comparison. There were no safety concerns with the use of recombinant activated FVII (rFVIIa) at doses/intervals as described in the product information. ${ }^{13}$

In HAVEN3, PWHAs without inhibitors aged 12 years or older on previous episodic treatment were randomly assigned to receive a subcutaneous maintenance dose of Emicizumab $1.5 \mathrm{mg} / \mathrm{kg}$ qw $(n=36)$ or $3.0 \mathrm{mg} / \mathrm{kg} \quad \mathrm{q} 2 \mathrm{w}$ $(n=35)$ or no prophylaxis $(n=18)$. The ABR was $1.5(95 \%$ CI: 0.9-2.5), 1.3 (95\% CI: 0.8-2.3) and 38.2 (95\% CI: 22.9-63.8) in the three groups, respectively $(p<0.001)$. The proportion of patients with no bleeds was 56 and $60 \%$ in patients on Emicizumab prophylaxis versus $0 \%$ in patients without prophylaxis. In an intraindividual comparison of 48 patients who received previous FVIII prophylaxis, a reduction of ABR of $68 \%$ was observed on Emicizumab prophylaxis $(p<0.001)$, suggesting a lower bleeding rate compared with their previous FVIII prophylactic regimen. Patients could continue on FVIII prophylaxis until the second loading dose. No thrombotic/TMA events occurred with concomitant FVIII treatment; most common adverse events were again, similar to older patients, injection-site reactions. ${ }^{5}$

A dosing regimen with a maintenance dose of $6 \mathrm{mg} / \mathrm{kg}$ $\mathrm{q} 4 \mathrm{w}$ in PWHAs with and without inhibitors showed similar efficacy with a low rate of treated bleeds (ABR: $2.4 ; 95 \% \mathrm{CI}$ : 1.4-4.3), and $56 \%$ of 41 patients reported no treated bleeds. ${ }^{9}$ Regimens have not been compared directly, but the mean ABR seems to be slightly higher in the $q 4 w$ regimen compared with q1w and $\mathrm{q} 2 \mathrm{w}$, maybe due to one outlier with a high bleeding rate (ABR: 32.07). 
Table 1 Summary of recommendations and level of agreement after Delphi survey

\begin{tabular}{|c|c|c|c|}
\hline & & Statement & $\begin{array}{l}\text { Agreement }^{\mathrm{a}} \\
N=13\end{array}$ \\
\hline General aspects & 1. & $\begin{array}{l}\text { Treatment with Emicizumab should be supervised by experienced } \\
\text { haemophilia centres. }\end{array}$ & $100 \%$ agreement \\
\hline \multirow[t]{3}{*}{ Patient education } & 2. & $\begin{array}{l}\text { Patients have to be educated before start of therapy with Emicizumab } \\
\text { (mode of action/impact on routine coagulation tests, route of } \\
\text { administration and dosing, storage, treatment strategies in case of } \\
\text { breakthrough bleeds or surgery) }\end{array}$ & $100 \%$ agreement \\
\hline & 3. & $\begin{array}{l}\text { An informative emergency card has to be issued to every patient on } \\
\text { Emicizumab }\end{array}$ & $100 \%$ agreement \\
\hline & 4. & $\begin{array}{l}\text { Every single administration of Emicizumab should be documented in a } \\
\text { patient diary }\end{array}$ & $100 \%$ agreement \\
\hline \multirow{7}{*}{$\begin{array}{l}\text { Management of } \\
\text { breakthrough } \\
\text { bleeds and surgery }\end{array}$} & 5. & $\begin{array}{l}\text { Each patient should have an emergency stock of FVIII or bypassing } \\
\text { agents at home for treatment of breakthrough bleeds }\end{array}$ & $\begin{array}{l}\text { 92.3\% agreement } \\
7.7 \% \text { limited agreement }\end{array}$ \\
\hline & 6. & $\begin{array}{l}\text { Bleeding treatment in PWHA with or without inhibitors should be } \\
\text { administered in relevant bleeds or significant injury }\end{array}$ & $\begin{array}{l}\text { 92.3\% agreement } \\
7.7 \% \text { limited agreement }\end{array}$ \\
\hline & 7. & $\begin{array}{l}\text { Not all non-severe bleeds need to be treated in patients receiving } \\
\text { Emicizumab prophylaxis. }\end{array}$ & $\begin{array}{l}\text { 92.3\% agreement } \\
7.7 \% \text { limited agreement }\end{array}$ \\
\hline & 8. & $\begin{array}{l}\text { For PWHA without inhibitors, clinically relevant breakthrough bleeds } \\
\text { should be treated with FVIII }\end{array}$ & $100 \%$ agreement \\
\hline & 9. & $\begin{array}{l}\text { For PWHA and inhibitors, rFVIla should be first-line treatment for } \\
\text { clinically relevant breakthrough bleeds. The use of aPCC in } \\
\text { doses }>100 \mathrm{U} / \mathrm{kg} \text { for more than } 24 \text { hours was associated with a risk of } \\
\text { thrombotic/TMA events. }\end{array}$ & $\begin{array}{l}\text { 92.3\% agreement } \\
7.7 \% \text { limited agreement }\end{array}$ \\
\hline & 10. & $\begin{array}{l}\text { For surgery in PWHA without inhibitors, the necessity, dose and } \\
\text { duration of FVIII replacement should be adapted to the surgical } \\
\text { procedure and the post-operative course. }\end{array}$ & $100 \%$ agreement \\
\hline & 11. & $\begin{array}{l}\text { For surgery in PWHA with inhibitors, first-line additional haemostatic } \\
\text { treatment is rFVIla. The need for additional treatment, dose and } \\
\text { duration of rFVIla replacement should be adapted to the surgical } \\
\text { procedure and the post-operative course. }\end{array}$ & $100 \%$ agreement \\
\hline \multirow{2}{*}{$\begin{array}{l}\text { Immune tolerance } \\
\text { induction (ITI) }\end{array}$} & 12. & In case of newly developed FVIII-inhibitors, ITI should be considered & $100 \%$ agreement \\
\hline & 13. & $\begin{array}{l}\text { ITI protocols combining FVIII to induce immune tolerance and Emi- } \\
\text { cizumab for prophylaxis have only been used in case series, therefore } \\
\text { no recommendation concerning indication, dose and duration of ITI } \\
\text { combined with Emicizumab prophylaxis can be made. }\end{array}$ & $\begin{array}{l}92.3 \% \text { agreement } \\
7.7 \% \text { limited agreement }\end{array}$ \\
\hline $\begin{array}{l}\text { Previously untreat- } \\
\text { ed patients (PUPs) }\end{array}$ & 14. & $\begin{array}{l}\text { Emicizumab is licensed for all age groups; however, licensure for } \\
\text { children is based on limited data. The decision to use Emicizumab in } \\
\text { small children, especially PUPs, has to be made on an individual base. }\end{array}$ & $\begin{array}{l}\text { 92.3\% agreement } \\
7.7 \% \text { limited agreement }\end{array}$ \\
\hline Elderly patients & 15. & $\begin{array}{l}\text { There are no general concerns to use Emicizumab in elderly patients } \\
\text { with HA. Individual risk factors and comorbidities must be taken into } \\
\text { account }\end{array}$ & $100 \%$ agreement \\
\hline \multirow[t]{2}{*}{ Laboratory tests } & 16. & $\begin{array}{l}\text { Emicizumab affects intrinsic pathway clotting-based laboratory } \\
\text { assays occurring after the first dose of Emicizumab and lasting up to } 6 \\
\text { months after the last dose }\end{array}$ & $100 \%$ agreement \\
\hline & 17. & $\begin{array}{l}\text { Tests to monitor FVIII replacement and FVIIlinhibitors as well as } \\
\text { Emicizumab concentration should be available }\end{array}$ & $100 \%$ agreement \\
\hline
\end{tabular}

Abbreviations: aPCC, activated prothrombin complex concentrate; FVIII, factor VIII; PWHA, patients with haemophilia A; TMA, thrombotic microangiopathy. a'Strong agreement' and 'agreement' are summarized as 'agreement'.

In HAVEN2, 88 PWHAs with inhibitors aged $<12$ years (or 12-17 years with $<40 \mathrm{~kg}$ body weight) with or without previous prophylactic bypassing agents (BPAs) were enrolled to receive Emicizumab prophylaxis with a maintenance dose of $1.5 \mathrm{mg} / \mathrm{kg} \mathrm{qw}(n=68), 3 \mathrm{mg} / \mathrm{kg} 2 \mathrm{qw}(n=10)$ or $6 \mathrm{mg} / \mathrm{kg}$ $\mathrm{q} 4 \mathrm{w}(n=10)$. In total, 18 patients were $\leq 2$ years old. The primary analysis revealed an ABR of 0.3 (95\% CI: $0.17-0.50)$, 0.2 (95\% CI: $0.03-1.72)$ and 2.2 (95\% CI: 0.69-6.81) in the groups treated $\mathrm{qw}, \mathrm{q} 2 \mathrm{w}$ and $\mathrm{q} 4 \mathrm{w}$, respectively. In the $\mathrm{q} 4 \mathrm{w}$ group, one patient developed neutralising anti-drug antibodies (ADA). Zero treated bleeds were reported in 76.9, 90 and $60 \%$ of patients, respectively. ${ }^{10}$ An interim 
analysis of 88 patients with inhibitors in the STASEY trial reported comparable bleeding rates and no further thrombotic events or other safety signals. ${ }^{14}$

\section{Patient Education}

2. Patients have to be educated before starting therapy with Emicizumab (mode of action/impact on routine coagulation tests, route of administration and dosing, storage, treatment strategies in case of breakthrough bleeds or surgery).

(a) Patients should be trained in subcutaneous injection.

(b) Patients or caregivers should regularly be trained in intravenous injection for emergency treatment with FVIII or BPAs.

(c) Inhibitor patients should be informed about the risk for developing thrombotic events or TMA when using aPCC in the presence of Emicizumab.

(d) Patients need to be informed about treatment strategies in case of bleeds or surgery.

(e) Patients should contact the treatment centre in case of bleeds to agree on treatment.

3. An informative emergency card has to be issued to every patient on Emicizumab.

(a) Contact details of the centre and the treatment strategy in case of bleeds/surgery have to be provided.

(b) In inhibitor patients the last inhibitor titre should be documented. Inhibitor testing should be performed at least every 6 months or according to clinical demands/ before surgical interventions.

(c) Information on Emicizumab interference with standard coagulation tests and precautions should be included.

4. Every single administration of Emicizumab should be documented in a patient diary.

(a) Additional treatment with clotting factor concentrates has to be documented.

(b) Data should be captured in registries, such as the German/Austrian/Swiss Haemophilia Registry or German Pediatric Haemophilia Research Database (GEPHARD), PedNet, etc.

\section{Management of Breakthrough Bleeds and Surgery}

5. Each patient should have an emergency stock of FVIII or BPAs at home for treatment of breakthrough bleeds.

(a) Patients should be provided with at least one to two doses of bleeding treatment medication, a bigger amount can be considered depending on the distance to the centre, travel activities or bleeding phenotype.

6. Bleeding treatment in PWHAs with and without inhibitors should be administered in relevant bleeds or significant injury.

(a) Joint bleeds, defined by pain, acute swelling and/or decreased range of motion. (b) Muscle bleeds/intracranial haemorrhage/retroperitoneal bleed/GI-bleed, etc.

7. Not all non-severe bleeds need to be treated in patients receiving Emicizumab prophylaxis.

(a) Minor bleeds or injuries (e.g., subcutaneous haematoma, nose bleeds, mucosal bleeds, small wounds) need individual evaluation.

(b) Tranexamic acid can be used locally or systemically.

8. For PWHAs without inhibitors, clinically relevant breakthrough bleeds should be treated with FVIII.

(a) Standard FVIII regimens according to bleeding type have been used without complications.

(b) The basal haemostatic activity of Emicizumab should be considered when treatment duration with additional FVIII is determined.

(c) If necessary, FVIII replacement can be monitored using a bovine chromogenic FVIII assay.

(d) After intensive treatment with FVIII, inhibitor testing should be performed using a chromogenic bovine Bethesda assay.

9. For PWHAs with inhibitors, rFVIIa should be first-line treatment for clinically relevant breakthrough bleeds. The use of aPCC in doses $>100 \mathrm{U} / \mathrm{kg}$ for more than 24 hours was associated with a risk of thrombotic/TMA events.

(a) Bleeding treatment with rFVIIa dosed according to prescription information was safe and clinically effective.

(b) In case of poor clinical response to rFVIIa, FVIII can be used in patients with low FVIII inhibitor activity.

(c) Recombinant porcine FVIII may be an option, however not licensed for patients with congenital haemophilia and inhibitors.

(d) aPCC should only be used if no other treatment options are available or effective.

(e) An initial aPCC dose of maximum $50 \mathrm{U} / \mathrm{kg}$ should not be exceeded. Lower doses (15-25 U/kg) have been described to be effective and safe in single cases.

(f) If a second dose of aPCC is required, the patient should be admitted to a hospital for surveillance (e.g., TMA, thrombotic events, control of bleed)

10. For surgery in PWHAs without inhibitors, the necessity, dose and duration of FVIII replacement should be adapted to the surgical procedure and the post-operative course.

(a) For minor surgery, additional FVIII treatment is not always necessary, but patients should be clinically monitored for occurrence of abnormal bleeding.

(b) Standard FVIII regimens according to bleeding risk of surgery have been used without complications.

(c) The basal haemostatic activity of Emicizumab should be considered when treatment duration with additional FVIII is determined.

(d) FVIII replacement should be monitored using a bovine chromogenic FVIII assay. 
(e) After intensive treatment with FVIII, inhibitor testing should be performed using a chromogenic bovine Bethesda assay.

(f) Currently, there is little experience with major surgery in PWHAs without inhibitors using Emicizumab prophylaxis.

11. For surgery in PWHAs with inhibitors, the first-line additional haemostatic treatment is rFVIIa. The need for additional treatment, dose and duration of $r F V I I$ a replacement should be adapted to the surgical procedure and the postoperative course.

(a) For minor surgery, not always additional BPA treatment is necessary, but patients should be clinically monitored for occurrence of abnormal bleeding.

(b) In major surgery, preventive treatment with rFVIIa should be used according to bleeding risk of surgery and clinical course.

(c) Inhibitor testing should be done before surgery to explore the possibility to treat the patient with FVIII in case of low or negative inhibitory activity.

(d) In case of inadequate efficacy of rFVIIa, recommendations for second-line treatment correspond to bleeding treatment [see point $10(a-f)$ ].

(e) If the use of aPCC at standard doses is planned to cover major surgery, Emicizumab has to be stopped for approximately 6 months upfront. Because of the long half-life of Emicizumab (4-5 weeks), interactions are expected for up to 6 months.

\section{Supporting Data}

\section{PWHAs without Inhibitors}

In PWHAs without inhibitors, additional FVIII replacement has been successfully used for treatment of breakthrough bleeds or surgery. The efficacy of FVIII replacement despite presence of Emicizumab is due to the lower binding affinity of Emicizumab to FIXa and FX with the effect that higher FVIII concentrations displace Emicizumab from binding sites. ${ }^{6} \mathrm{~A}$ non-additive effect of FVIII in combination with Emicizumab has also been shown in thrombin generation assays. ${ }^{15}$ No thrombotic/TMA events occurred with additional FVIII replacement, ${ }^{5}$ therefore there are no safety concerns for the use of FVIII in standard dosing regimens. Not all bleeds occurring under Emicizumab prophylaxis need to be treated. In HAVEN3, a total ABR of 2.5 (95\% CI: 1.6-3.9) in patients treated with $1.5 \mathrm{mg} / \mathrm{kg}$ qw and $2.6(95 \% \mathrm{CI}: 1.6-4.3)$ in the group treated with $3 \mathrm{mg} / \mathrm{kg}$ q2w was reported with only 1.5 (95\%Cl: $0.9-2.5)$ and 1.3 (95\% CI: 0.8-2.3) treated bleeds, respectively. $^{5}$

For minor surgical procedures, additional FVIII replacement is not always necessary. In HAVEN3, 50 procedures in 30 patients have been performed, of those 46 were minor surgeries, including dental $(n=17)$, oesophagogastroduodenoscopy or colonoscopy $(n=14)$, and orthopaedic $(n=10)$ procedures. In total, 28 (61\%) of these procedures were performed without additional preventive FVIII replacement with only one bleed in a patient with tooth extraction. A total of
18 patients received preventive FVIII replacement, 14 for less than 24 hours. Overall, three patients experienced bleeds; however, no patient required treatment for $>72$ hours. Four patients with major orthopaedic surgery received preventive FVIII infusions for 14 to 18 days without bleeding complications. No thrombotic event and no de novo inhibitors occurred. $^{16}$

\section{PWHAs with Inhibitors}

In HAVEN1, two thrombotic and three TMA events occurred in patients treated with additional aPCC $>100 \mathrm{U} / \mathrm{kg}$ for $>24$ hours. ${ }^{4}$ An additional TMA was reported post-marketing. ${ }^{12}$ Interaction of aPCC with Emicizumab leads to excessive thrombin generation (synergistic effect), because aPCC contains FIXa and FX, the substrate for Emicizumab. ${ }^{17}$ Lower doses of aPCC (15-25 U/kg) have been shown to be effective in vitro and in vivo in a patient with a spontaneous psoas bleed. ${ }^{18}$ In vitro measurements of ROTEM and thrombin generation assays suggest that even lower doses of aPCC can be effective in PWHAs with inhibitors under Emicizumab, which has been shown by spiking experiments, showing similar effect of aPCC $10 \mathrm{U} / \mathrm{kg}$ in Emicizumab-treated haemophilia A (HA) as $100 \mathrm{U} /$ $\mathrm{kg}$ in Emicizumab-untreated HA. ${ }^{19}$ In the HAVEN study program, 210 bleeds have been treated with rFVIIa with no occurrence of thrombosis or TMA, also at high doses. ${ }^{13}$

Not all bleeds in PWHAs with inhibitors under Emicizumab prophylaxis need to be treated with additional BPA. In HAVEN1, the total ABR was 5.5 (95\% CI: 3.6-8.6) and 2.9 (95\% CI: $1.7-5.0)$ in treated bleeds. In total, $47 \%$ of bleeds could be treated with a single rFVIIa treatment. ${ }^{4}$ If rFVIIa is not effective for bleeding treatment or surgery, in PWHAs with low-titre FVIII inhibitors, FVIII has been used successfully for up to 6 days. ${ }^{20}$ Theoretically, also recombinant porcine FVIII could be an option in refractory bleeding; however, it is only licensed for acquired HA. ${ }^{21}$

In HAVEN1 and 2, 19 minor surgeries [13 central venous access devices (CVADs), 6 tooth extractions, 9 and 4 without additional BPA treatment, respectively] have been reported. In none of the CVAD procedures bleeding occurred, but in $50 \%$ of tooth extraction (in one with and one without additional preventive BPA). ${ }^{22}$ Further nine cases with surgical procedures experienced a low bleeding frequency with one to six doses of rFVIIa $(90 \mu \mathrm{g} / \mathrm{kg}$ ) or FVIII ( $100 \mathrm{IU} / \mathrm{kg}$, in the case of low-titre inhibitor) or observation. ${ }^{23}$ Altogether, within the HAVEN study program 215 minor surgeries have been performed in 115 patients (64 dental, 34 CVADs, 30 endoscopic, 25 joint, and 62 other procedures). Of the 141 (65.6\%) minor surgeries/procedures managed without prophylactic coagulation factor, 128/141 (90.8\%) did not result in treated post-operative bleeds. In 14/64 dental procedures, treated post-operative bleeds were reported, in 9/42 (21.4\%) in patients without and 5/22 (2.7\%) in patients with prophylactic coagulation factor replacement. ${ }^{24}$

In addition, 19 major surgeries have been performed in 19 patients, of which 5 were joint arthroplasties, 4 synovectomies and 9 other procedures. In 16/19 major surgeries, prophylactic replacement of FVIII or rFVIIa was given resulting in 1 treated and 2 untreated post-operative bleeds. 
Three procedures were performed without prophylactic replacement therapy without bleeding complications. No thromboembolic events occurred. ${ }^{22,24}$ In one of the above patients with the treated post-operative bleed, a right-hip replacement was performed with the coverage of rFVIIa and lacking response (developed a right-thigh haematoma and experienced dropping haemoglobin levels). Since the inhibitor titre was low, FVIII treatment could be safely used in this patient for several days until anamnestic response occurred. ${ }^{20}$ In two further cases of the HAVEN trials, major surgery was conducted with the use of rFVIIa safely: a total hip arthroplasty ${ }^{25}$ and knee replacement. ${ }^{26}$

\section{Immune Tolerance Induction}

12. In case of newly developed FVIII inhibitors, ITI should be considered.

(a) Successful ITI offers the possibility to treat bleeds and to cover surgery and bleeds with FVIII, as well as access to gene therapy.

(b) Standard ITI protocols, e.g., Bonn protocol, have been proven to be effective (60-80\%) and safe. ${ }^{27-29}$

(c) To prevent breakthrough bleeds, ITI should be started immediately after inhibitor confirmation.

(d) If the start of ITI has to be postponed, prophylactic treatment with Emicizumab can be considered.

(e) In patients on ITI experiencing frequent breakthrough bleeds, a prophylactic approach using BPA or Emicizumab can be considered.

(f) In patients having been treated with Emicizumab $<6$ months, the use of aPCC as a prophylactic agent during ITI has to be avoided.

13. ITI protocols combining FVIII to induce immune tolerance and Emicizumab for prophylaxis have only been used in case series, therefore no recommendation concerning indication, dose and duration of ITI combined with Emicizumab prophylaxis can be made.

(a) No controlled study data are available yet on ITI with FVIII in combination with Emicizumab.

(b) ITI courses combined with Emicizumab should be documented in studies or registries.

(c) The peak inhibitor titre should be taken into consideration when choosing the FVIII dose.

(d) During ITI combined with Emicizumab prophylaxis, FVIII and FVIII inhibitors have to be monitored using chromogenic bovine FVIII assays.

(e) How to maintain FVIII tolerance after successful ITI is not clear. If patients remain on Emicizumab, the necessity, amount and duration of additional FVIII application is not known.

\section{Supporting Data}

Eradication of inhibitors using ITI therapy is an effective, safe and proven strategy to eradicate inhibitors in PWHAs. ${ }^{27-29}$ The Bonn protocol ${ }^{30}$ using FVIII at doses of 100 to $150 \mathrm{IU} / \mathrm{kg}$ twice daily and BPA prophylaxis in case of breakthrough bleeds is standard of care in Germany. Even in the era of
Emicizumab, national guidelines and experts recommend the use of ITI. ${ }^{31-34}$ Successful inhibitor eradication allows safer management of bleeds and surgery, sustain patient's or parent's ability to infuse intravenous clotting factor and access to future therapeutic options (e.g., gene therapy). The availability of Emicizumab allows postponing ITI and offering effective bleeding prophylaxis during ITI. Experience with ITI for inhibitor eradication and concomitant Emicizumab prophylaxis is limited. Recently, the first report of seven paediatric inhibitor patients aged 21 months to 12 years undergoing the Atlanta protocol, i.e., 100 IU FVIII/ $\mathrm{kg}$ threetimes per week and Emicizumab prophylaxis, has been published. Inhibitor titres decreased in all patients. Three of the seven patients achieved negative inhibitor titres $[<0.6$ chromogenic Bethesda units $(\mathrm{CBU}) / \mathrm{mL}]$ and two patients achieved a normal FVIII recovery ( $>66 \%$ ). There were nine bleeding episodes in four patients and no thrombotic events. ${ }^{35}$ Complete success has not been published yet. To date, all patients still have been on the ITI FVIII dose.

Questions remain on the optimal FVIII dose /ITI protocol, the approach for tapering down FVIII ITI dosing and how to maintain tolerance after successful ITI.

ITI courses combined with Emicizumab and the treatment strategy should be documented in studies or registries (MOTIVATE study, PedNet, ObsITI, etc.).

\section{Previously Untreated Patients}

14. Emicizumab is licensed for all age groups; however, licensure for children is based on limited data. The decision to use Emicizumab in small children, especially PUPs, has to be made on an individual basis.

\section{Supporting Data}

There are no data on the efficacy, pharmacokinetic/pharmacodynamic and safety of Emicizumab administration in newborns with HA so far. The equilibrium of the coagulation system of the newborns (coagulation, anticoagulation, fibrinolysis) differs from older children. Since haemostatic efficacy of Emicizumab is dependent on FIX activity, it is important to consider that concentrations of FIX are reduced at birth and reach normal values at the age of approximately 6 months. Even in older children, FIX levels might be physiologically reduced. Whether prevention of intracerebral bleeds can be achieved with Emicizumab prophylaxis started in early life needs to be explored in further studies.

In the frame of the HAVEN2 trial, only five infants with severe HA and inhibitors ( 1 month up to $<2$ years) have been included. Another three haemophilia patients without inhibitors aged $<3$ years have been treated in the HOHOEMI study $^{36}$ without any safety concerns. In the frame of the HAVEN studies in children $<12$ years of age, no differences of efficacy have been observed between the different age groups. The steady state plasma trough concentrations of Emicizumab were comparable in adult and paediatric patients older than 6 months. In addition, the adverse events were similar in type to those observed in adult HA patients. ${ }^{10}$ 
A single-centre experience reported the prophylactic use of Emicizumab in 11children with inhibitors (median age: 26 months). Prophylaxis was judged to prevent patients from haemarthrosis and spontaneous bleeds. Four out of 11 patients were treated with rFVIIa occasionally. Three minor surgeries were performed, one complicated by major bleeding. The authors emphasize that bleeds occurred in those patients with the lowest thrombin generation results. ${ }^{37}$

In general, there are no data available on the treatment strategy in PUPs (Emicizumab stand-alone prophylaxis or Emicizumab prophylaxis combined with the episodic prophylactic administration of FVIII). Studies are urgently needed and are currently planned (FREE-study, Finding the Response to Early Emicizumab in PUPs $<2$ years of age). All PUP data should be captured in the GEPHARD registry.

\section{Elderly Patients}

15. There are no general concerns to use Emicizumab in elderly patients with HA. Individual risk factors and co-morbidities must be taken into account.

\section{Supporting Data}

The HAVEN study program also included elderly patients. Median age was 28 years (range: 12-75), 38 years (range: 13-77), 39 years (range:14-68) in HAVEN 1, 3 and 4, respectively. Exact numbers of patients aged $>65$ years are not published. ${ }^{4,5,9}$

Altogether, at end of September 2019, more than 5,400 patients have been treated with Emicizumab worldwide. Apart from the 4 cases of TMA in patients treated with additional high doses of BPA, 9 thrombotic events (not TMAs) occurred, 2 in the HAVEN 1 study with high doses of BPA and 7 without additional BPA or low doses. Most patients had additional risk factors like arterial hypertension and previous venous thromboembolism. ${ }^{12}$

There is no evidence that age itself is a risk factor, but patients should be evaluated for cardiovascular risk factors and decision to prescribe Emicizumab to patients with risk factors should be based on individual benefit/risk evaluation.

\section{Laboratory Tests}

16. Emicizumab affects intrinsic pathway clotting based laboratory assays occurring after the first dose of Emicizumab and lasting up to 6 months after the last dose.

(a) Activated partial thromboplastin time (aPTT; shortened) and all aPTT-based one-stage clotting assays (e.g., FVIII-XII, classical [Nijmegen modified] Bethesda assay, protein C/protein S activity, activated protein Cresistance) are influenced by Emicizumab and cannot be interpreted during the use and up to 6 months after discontinuation of Emicizumab. ${ }^{38,39}$

(b) The following tests are not influenced by Emicizumab: thrombin time, prothrombin time based one-stage tests for single factor activity, chromogenic factor assays (except for FVIII), chromogenic FVIII assay with bovine components, immune-based assays (ELISA; turbidimetric methods) and Bethesda assay with the bovine chromogenic FVIII assay.

17. Tests to monitor FVIII replacement and FVIII inhibitors as well as Emicizumab concentration should be available in centres treating PWHAs with Emicizumab.

(a) For monitoring FVIII replacement in the presence of Emicizumab, a chromogenic test with bovine components has to be used.

(b) Inhibitor testing has to be performed with a bovine chromogenic test as well. Inhibitory activity is expressed in $\mathrm{CBU} / \mathrm{mL}$.

(c) Monitoring of Emicizumab concentration should be used with the aim to detect lacking therapy adherence or neutralising ADAs. Fully neutralising ADAs are captured with measuring a prolonged aPTT, but partially neutralising ADAs need more sensitive tests.

(d) If aPCC at standard doses is intended to be used, sensitive tests to monitor Emicizumab concentration can be useful to exclude relevant concentrations. Because of the long half-life of Emicizumab, relevant concentrations can be expected for up to 6 months after the last dose of Emicizumab.

(e) Emicizumab concentration can be monitored using a chromogenic test with human components or a diluted one-stage clotting assay calibrated against Emicizumab.

(f) We recommend monitoring of Emicizumab concentration 1 week after the last loading dose and then every 3 months for a year, then every 6 to 12 months or in case of lacking efficacy. Expected trough levels in the steady state with the $1.5 \mathrm{mg} /$ week dosing regimens are 25 to $75 \mu \mathrm{g} / \mathrm{L}{ }^{40}$

(g) For monitoring FVIII replacement or for FVIII inhibitor testing in the presence of Emicizumab, a chromogenic test with bovine components can be used.

\section{Supporting Data}

Development of ADAs against Emicizumab is rare. In the HAVEN study program, 14 out of 398 patients were tested positive for ADA, in 7 of whom the antibodies were transient, and 3 patients $(0.75 \%)$ had neutralizing antibodies with reduction of efficacy including one patient with a fully neutralizing ADA resulting in lacking efficacy including repeated break-through bleeds. ${ }^{41}$

For monitoring Emicizumab concentration in plasma, a chromogenic test with human components showed good linearity within the expected plasma concentrations (5-150 $\mu \mathrm{g} / \mathrm{mL}$ ). Also, a one-stage clotting assay with diluted patient plasma (1:80) has been validated for measuring Emicizumab concentration. For both tests, Emicizumab should be used as a calibrator. ${ }^{38-40}$ FVIII measurement is not influenced by Emicizumab if a chromogenic test with bovine FIXa and FX as reagents is used. ${ }^{38}$

\section{Conclusion}

This studyis designed to support haemophilia physicians using Emicizumab in PWHA. Deliberate education 
of patients and medical staff is required as well as adjusted strategies in case of break-through bleeding and surgery. With emerging further experience and clinical trials, these recommendations need to be updated at regular intervals.

\section{Author Contributions}

K.H. and C.E.E. performed the literature search and wrote the first draft of the manuscript. K.H., C.B. and C.E.E. drafted the recommendations. All authors took part in the discussion of recommendations, the Delphi survey and contributed to manuscript writing. All authors critically reviewed the final manuscript and approved it for publication.

\section{Conflict of Interest}

K.H. received honoraria for advisory boards, travel grants and/or speaker fees from Bayer, Biotest, Chugai, CSL Behring, NovoNordisk, Pfizer, Roche, Shire/Takeda and Sobi, and unrestricted research grants from Bayer, CSL Behring and Pfizer. M.A. received honoraria for advisory boards, travel grants, speaker fees and/or research funding from Biogen, Biotest, Novo Nordisk, Roche, Shire and Sobi. C.B. received honoraria for advisory boards, travel grants, research funding and/or speaker fees from Bayer, Biotest, CSL Behring, NovoNordisk, Pfizer, Roche, Shire/Takeda and Sobi. C.B. is investigator in the STASEY Trial (Roche). C.B. and C.K. are principal investigators of the German Pediatric Haemophilia Research Database (GEPHARD). GEPHARD is funded by manufacturers of haemophilia drugs. S.Ha. received honoraria for advisory boards or speaker's fees from Bayer, Baxalta/Shire/Takeda, Biotest, CSL Behring, Novartis, Novo Nordisk, Octapharma and Pfizer, and research grants from Bayer, Shire/Takeda, Biotest, CSL Behring, Novo Nordisk, Octapharma and Pfizer. S.He. received honoraria for advisory boards or speaker's fees from Bayer, Shire/Takeda, CSL Behring, Biotest, NovoNordisk, Pfizer, Roche and Sobi. R.K. has acted as a consultant, received speaker's fees and/or research funding from Bayer Healthcare, Biomarin, Biotest, CSL Behring, Grifols, Octapharma, Pfizer, NovoNordisk, Sanofi, Shire/Takeda, Sobiand Roche/ Chugai. C.K. received honoraria for advisory boards, travel grants and/or speaker fees from Bayer, BFSH, Biotest, Bioverativ, CSL Behring, MSD, NovoNordisk, Pfizer, Roche, Shire and Sobi, and unrestricted research grants from the European Union, FP7 IMI, DFG, BMBF, FSKK-Foundation, Abbvie, Bayer, Biotest, Bioverativ, CSL Behring, Gilead, Intersero, Jansen, NovoNordisk, PENTA Foundation, Pfizer, Roche/Chugai, Shire and Sobi. K.K. received grants, travel support and/or lecture fees from Bayer, Biotest, CSL Behring, NovoNordisk, Roche, Sobi, Shire/Takeda and honoraria for advisory board from Shire/Takeda. C.M. has received personal honoraria (consultancy, speaker, chair) from Bayer, Biotest, CSL Behring, NovoNordisk, Roche; fees to the institution for study participation from Bayer, Shire/Takeda, Biotest, CSL Behring, NovoNordisk, Sobi; unrestricted grants to institution from Biotest, CSL Behring; travel support from Bayer, Biotest, CSL Behring, NovoNordisk. J.O. received reimbursement for attending symposia/congresses and/or honoraria for speaking and/or honoraria for consulting, and/or funds for research from Bayer, Biogen Idec, Biotest, Chugai, CSL-Behring, Freeline, Grifols, Novo Nordisk, Octapharma, Pfizer, Roche, Sparks, Swedish Orphan Biovitrum and Takeda. W.S. has received honoraria and research funding from Biotest, Octapharma, Pfizer, Sobiand Shire/Takeda. W.S. has been a member of expert panels and advisory committees from NovoNordisk, Roche, Sobi and Shire/Takeda. C.W. is an employee at Werlhof-Institut, received honoraria from Bayer, Biotest, CSL-Behring, LFB, Novo Nordisk, Pfizer, Shire and Sobi, and acted as a consultant in advisory boards for Bayer, CSLBehring, Novo Nordisk, Pfizer, Shire and Sobi. C.E.E has acted as a consultant, received speaker's fees and/or research funding from Bayer Healthcare, Biotest, CSL Behring, Grifols, Octapharma, NovoNordisk, Shire/Takeda, Sobi, Roche/Chugai, Alnylam and Kedrion.

\section{References}

1 Kitazawa T, Igawa T, Sampei Z, et al. A bispecific antibody to factors IXa and $X$ restores factor VIII hemostatic activity in a hemophilia A model. Nat Med 2012;18(10):1570-1574

2 Shima M, Hanabusa H, Taki M, et al. Factor VIII-mimetic function of humanized bispecific antibody in hemophilia A. N Engl J Med 2016;374(21):2044-2053

3 Uchida N, Sambe T, Yoneyama K, et al. A first-in-human phase 1 study of ACE910, a novel factor VIII-mimetic bispecific antibody, in healthy subjects. Blood 2016;127(13):1633-1641

4 Oldenburg J, Mahlangu JN, Kim B, et al. Emicizumabprophylaxis in hemophilia A with inhibitors. N Engl J Med 2017;377(09): 809-818

5 Mahlangu J, Oldenburg J, Paz-Priel I, et al. Emicizumabprophylaxis in patients who have hemophiliaA without inhibitors. $\mathrm{N}$ Engl J Med 2018;379(09):811-822

6 Lenting PJ, Denis CV, Christophe OD. Emicizumab, a bispecific antibody recognizing coagulation factors IX and X: how does it actually compare to factor VIII? Blood 2017;130(23):2463-2468

7 Moher D, Liberati A, Tetzlaff J, Altman DG; PRISMA Group. Preferred reporting items for systematic reviews and metaanalyses: the PRISMA statement. PLoS Med 2009;6(07):e1000097

8 Hasson F, Keeney S, McKenna H. Research guidelines for the Delphi survey technique. J Adv Nurs 2000;32(04):1008-1015

9 Pipe SW, Shima M, Lehle M, et al. Efficacy, safety, and pharmacokinetics of emicizumab prophylaxis given every 4 weeks in people with haemophilia A (HAVEN 4): a multicentre, open-label, nonrandomised phase 3 study. Lancet Haematol 2019;6(06):e295e305

10 Young G, Liesner R, Sidonio R, et al. Emicizumabprophylaxis provides flexible and effective bleed control in children with hemophilia A with inhibitors: results from the HAVEN 2 study. Blood 2018;132(Suppl 1):632-632

11 Mancuso ME, Callaghan MU, Kruse-Jarres R, et al. Emicizumabprophylaxis in adolescent/adult patients with hemophilia $\mathrm{A}$ previously receiving episodic or prophylactic bypassing agent treatment: updated analyses from the HAVEN 1 study. Blood 2017;130(Suppl 1):1071-1071

12 Genentech U. Hemlibra global safety report for health care professionals. Available at: https://www.emicizumabinfo.com/hcp/emicizumab-safety-information.html. Accessed October 18, 2019

13 Levy GG, Asikanius E, Kuebler P, Benchikh El Fegoun S, Esbjerg S, Seremetis S. Safety analysis of rFVIIa with emicizumab dosing in 
congenital hemophilia A with inhibitors: experience from the HAVEN clinical program. J ThrombHaemost 2019;17(09):1470-1477

14 Jimenez-Yuste V, Klamroth R, Castaman G, Ozelo M, Aout M, Meier O, Santagostino E. A single-arm, multicentre, open-label, phase III clinical trial to evaluate the safety and tolerability of prophylactic emicizumab in persons with haemophilia A (PwHA) with FVIII inhibitors (STASEY): interim analysis results. Oral Abstract OC 60.3.Paper presented at: International Society on Thrombosis and Haemostasis (ISTH) Congress, Melbourne, Australia, July 6-10, 2019. (Res PractThrombHemost2019;3(1))

15 Bravo MI, Raventós A, Pérez A, Costa M, Willis T. Plasma-derived FVIII/VWF combined with emicizumab in hemophilia A plasma with and without inhibitor has a non-additive effect on in vitro thrombin generation Oral Abstract OC 53.3. Paper presented at: the International Society on Thrombosis and Haemostasis (ISTH) Congress, Melbourne, Australia, July 6-10, 2019.(Research and Practice in Thrombosis and Haemostasis, 2019. 3(S1))

16 Santagostino E, Parnes A, Dhalluin C, et al. Surgical procedures in persons with haemophilia A (PWHA) without inhibitors receiving emicizumab - experience from the HAVEN 3 study. Haemophilia 2019;25(S1):33

17 Hartmann R, Feenstra T, Valentino L, Dockal M, Scheiflinger F. In vitro studies show synergistic effects of a procoagulant bispecific antibody and bypassing agents. J ThrombHaemost 2018

18 Dargaud Y, Lienhart A, Janbain M, Le Quellec S, Enjolras N, Negrier C. Use of thrombin generation assay to personalize treatment of breakthrough bleeds in a patient with hemophilia and inhibitors receiving prophylaxis with emicizumab. Haematologica 2018; 103(04):e181-e183

19 Furukawa S, Nogami K, Matsumoto T, Kasai R, Shima M. Preanalysis for hemostatic effect of bypassing agents in hemophilia a patients with inhibitors under the emicizumab prophylaxis by global assays. Blood 2018;132(Suppl 1):3793-3793

20 Santagostino E, Mancuso ME, Novembrino C, Solimeno LP, Tripodi A, Peyvandi F. Rescue factor VIII replacement to secure hemostasis in a patient with hemophilia $A$ and inhibitors on emicizumab prophylaxis undergoing hip replacement. Haematologica 2019; 104(08):e380-e382

21 Kruse-Jarres R, St-Louis J, Greist A, et al. Efficacy and safety of OBI-1, an antihaemophilic factor VIII (recombinant), porcine sequence, in subjects with acquired haemophilia A. Haemophilia 2015;21(02): $162-170$

22 Kruse-Jarres R, Callaghan MU, Croteau SE, et al. Surgical experience in two multicenter, open-label phase 3 studies of emicizumab in persons with hemophilia A with inhibitors (HAVEN 1 and HAVEN 2). Blood 2017;130(Suppl 1):89-89

23 Zimowski KL, Batsuli GM, Reding MT, et al. Maintaining perioperative hemostasis in patients with severe hemophiliaA and inhibitors receiving emicizumab prophylaxis. Blood 2018;132(Suppl 1):635-635

24 Santagostino E, Oldenburg J, Chang T, et al. Surgical experience from four phase III studies (HAVEN 1-4) of emicizumab in persons with haemophilia A (PwHA) with or without FVIII inhibitors. Oral Abstract OC 60.1. Paper presented at: the International Society on Thrombosis and Haemostasis (ISTH) Congress, Melbourne, Australia, July 6-10, 2019.(Research and Practice in Thrombosis and Haemostasis, 2019. 3(S1):1-228)

25 Seaman CD, Ragni MV. Emicizumab use in major orthopedic surgery. Blood Adv 2019;3(11):1722-1724
26 Kizilocak H, Yukhtman CL, Marquez-Casas E, Lee J, Donkin J, Young G. Management of perioperative hemostasis in a severe hemophilia A patient with inhibitors on emicizumab using global hemostasis assays. Ther Adv Hematol 2019;10:2040620719860025

27 Oldenburg J, Schwaab R, Brackmann HH. Induction of immune tolerance in haemophilia A inhibitor patients by the 'Bonn Protocol': predictive parameter for therapy duration and outcome. Vox Sang 1999;77(Suppl 1):49-54

28 Kreuz W, Ehrenforth S, Funk M, et al. Immune tolerance therapy in paediatric haemophiliacs with factor VIII inhibitors: 14 years follow-up. Haemophilia 1995; 1(01):24-32

29 Brackmann HH, Lenk H, Scharrer I, Auerswald G, Kreuz W. German recommendations for immune tolerance therapy in type A haemophiliacs with antibodies. Haemophilia 1999;5(03):203-206

30 Brackmann HH, Gormsen J. Massive factor-VIII infusion in haemophiliac with factor-VIII inhibitor, high responder. Lancet 1977;2(8044):933

31 Collins PW, Liesner R, Makris M, et al. Treatment of bleeding episodes in haemophilia A complicated by a factor VIII inhibitor in patients receiving emicizumab. Interim guidance from UKHCDO Inhibitor Working Party and Executive Committee. Haemophilia 2018;24(03):344-347

32 Oldenburg J, Young G, Santagostino E, Escuriola Ettingshausen C. The importance of inhibitor eradication in clinically complicated hemophilia A patients. Expert Rev Hematol 2018;11(11):857-862

33 Santagostino E, Young G, Escuriola Ettingshausen C, JimenezYuste V, Carcao M. Inhibitors: a need for eradication? Acta Haematol 2019;141(03):151-155

34 MASAC Recommendations. Available at: https://www.hemophilia. org/Researchers-Healthcare-Providers/Medical-and-ScientificAdvisory-Council-MASAC/MASAC-Recommendations/Recommendation-on-the-Use-and-Management-of-Emicizumab-kxwhHemlibra-for-Hemophilia-A-with-and-without-Inhibitors. Accessed 26 August, 2019

35 Batsuli G, Zimowski KL, Tickle K, Meeks SL, Sidonio RF Jr. Immune tolerance induction in paediatric patients with haemophilia $A$ and inhibitors receiving emicizumab prophylaxis. Haemophilia 2019;25(05):789-796

36 Shima M, Nogami K, Nagami S, et al. A multicentre, open-label study of emicizumab given every 2 or 4 weeks in children with severe haemophilia A without inhibitors. Haemophilia 2019;25 (06):979-987

37 Barg AA, Avishai E, Budnik I, et al. Emicizumab prophylaxis among infants and toddlers with severe hemophilia A and inhibitors-a single-center cohort. Pediatr Blood Cancer 2019;66(11):e27886

38 Adamkewicz JI, Chen DC, Paz-Priel I. Effects and interferences of emicizumab, a humanised bispecific antibody mimicking activated factor VIII cofactor function, on coagulation assays. ThrombHaemost 2019;119(07):1084-1093

39 Müller J, Pekrul I, Pötzsch B, Berning B, Oldenburg J, Spannagl M. Laboratory monitoring in emicizumab-treated persons with hemophilia A. ThrombHaemost 2019;119(09):1384-1393

40 Türkantoz H, Varnholt D, Tiede A. Monitoring of emicizumab (ACE910): comparison between clotting and chromogenic assay. 2019Haemophilia25(S1):98-99

41 Paz-Priel I, Chang T, Asikanius E, et al. Immunogenicity of emicizumab in people with hemophiliaA (PwHA): results from the HAVEN 1-4 studies. Blood 2018;132(Suppl 1):633-633 